\title{
HBx-induced miR-21 suppresses cell apoptosis in hepatocellular carcinoma by targeting interleukin-12
}

\author{
DIAN YIN $^{1 *}$, YILANG WANG $^{1 *}$, WENLI SAI ${ }^{2}$, LIANG ZHANG $^{1}$, YAJUN MIAO $^{1}$, \\ LILI CAO $^{1}$, XIAOLU ZHAI $^{1}$, XIU FENG $^{1}$ and LI YANG $^{1}$ \\ ${ }^{1}$ Department of Oncology, The First People's Hospital of Nantong; ${ }^{2}$ Research Center of Clinical Medicine, \\ Affiliated Hospital of Nantong University, Nantong, Jiangsu 226001, P.R. China
}

Received March 3, 2016; Accepted April 19, 2016

DOI: $10.3892 /$ or.2016.5026

\begin{abstract}
Hepatitis B virus (HBV) X protein (HBx) plays a key role in the initiation and progression of HBV infection-induced hepatocellular carcinoma (HCC). Oncogenic microRNA-21 (miR-21) can be modulated by HBx protein in HCC. However, critical regulator genes in the pathway of HBx-induced miR-21 in HCC remain unclear. This study aimed to investigate the role of HBx-induced miR-21 in the apoptosis of HCC cells. In the study, interleukin-12 (IL-12) was demonstrated as a direct target of miR-21 by dual-luciferase report assay, and miR-21 was highly expressed in HCC cells (HepG2 and HepG2 2.2.15) compared to L02 cells, but IL-12 was weakly expressed as detected by real-time quantitative PCR (RT-qPCR). Furthermore, miR-21 mimics, inhibitor, HBx-targeted siRNA, and the HBx overexpression vector $(\mathrm{pHBx})$ were used to observe the regulatory effects of HBx-induced miR-21 via IL-12, and cell apoptosis was assessed. The results showed that overexpression of $\mathrm{HBx}$ resulted in the inhibition of IL-12. A high level of miR-21 resulted in a significant increase in proliferation and a decrease in IL-12 expression. Inhibition of miR-21 resulted in a significant increase in apoptosis and increased IL-12 expression. The results suggest that HCC cell apoptosis was suppressed at least partially through HBx-induced miR-21 by targeting IL- 12 .
\end{abstract}

\section{Introduction}

Chronic infection by hepatitis B virus (HBV) is strongly associated with the initiation and progression of hepatocellular carcinoma $(\mathrm{HCC})(1,2)$, and HBV X protein $(\mathrm{HBx})$ is a major risk factor in the molecular pathogenesis of $\mathrm{HBV}$-related HCC $(3,4)$. HBx is encoded by the HBV genome, and it is

Correspondence to: $\mathrm{Dr} \mathrm{Li}$ Yang, Department of Oncology, The First People's Hospital of Nantong, 6 North Hai'er Xiang Road, Nantong, Jiangsu 226001, P.R. China

E-mail: ntyyzlkyl@163.com

*Contributed equally

Key words: miR-21, hepatitis B virus X, hepatocellular carcinoma, interleukin-12, apoptosis required for mammalian hepadnavirus infectivity and replication (5). In human hepatocytes, HBx has multiple molecular functions by interacting with different transcription factors and modulating numerous cellular signaling pathways (6-8).

microRNAs (miRNAs) are a class of endogenous small RNAs 19 to 23 nucleotides (nt) in length, which have been studied as regulators of gene expression in biological processes, including cell development, differentiation, apoptosis, and proliferation (9). Recent studies have shown that HBx protein can induce the differential expression of miRNAs, such as miR-520 (10), miR-145, miR-222, miR-21 (11) and miR-146 (12). Oncogenic miR-21 was reported as an important miRNA induced by $\mathrm{HBx}$, and promotes cell proliferation (13) and transformation (14), but the related mechanism is not yet fully elucidated.

Interleukin-12 (IL-12) was discovered as a "natural killer-stimulating factor' and a 'cytotoxic lymphocyte maturation factor' $(15,16)$. IL-12 is produced by monocytes, macrophages, B cells and dendritic cells. IL-12 is known as a $\mathrm{T}$ cell-stimulating factor, which can stimulate the production of interferon- $\gamma$ (IFN- $\gamma$ ) and tumor necrosis factor- $\alpha$ (TNF- $\alpha$ ) from $\mathrm{T}$ cells and natural killer (NK) cells, and favors the differentiation of naive $\mathrm{CD}^{+} \mathrm{T}$ cells into mature $\mathrm{Th} 1$ cells (17). Studies have reported that IL-12 as a cytokine has antitumor therapeutic activities, and it has been shown to inhibit tumorigenesis and induce regression of established tumors. IL-12 promotes the effective destruction of cancer cells by inducing proliferation of NK and T cells, and IL-12 enhances the generation and activity of cytotoxic T lymphocytes (CTLs) $(18,19)$. In addition, several other mechanisms of IL-12 strongly contribute to antitumor activities $(20,21)$, and the antitumor activity of IL-12 can be improved by its combination with various therapeutics $(22,23)$.

In this study, we first report that IL-12 was regulated by HBx-induced miR-21 in human hepatocytes, especially in $\mathrm{HCC}$ cells. The study aimed to reveal the role of $\mathrm{HBx}$-induced miR-21 and IL-12 in HCC biology.

\section{Materials and methods}

Cell culture and transfection. Human HCC cell lines HepG2 and HepG2 2.2.15, normal liver L02 cells and human embryonic kidney 293 (HEK293) cells were maintained in Dulbecco's 
Table I. Sequences of the RT-qPCR primers.

\begin{tabular}{ll}
\hline Genes & \multicolumn{1}{c}{ Sequences $\left(5^{\prime}-3^{\prime}\right)$} \\
\hline HBx & F: GTCTGTGCCTTCTCATCTG \\
& R: GGTCGGTCGTTGACATTG \\
IL-12 & F: CTCCTCCTTGTGGCTACC \\
& R: TGAAGGCATGGGAACATTC \\
$\beta$-actin & F: AATCGTGCGTGACATTAAG \\
& R: GAAGGAAGGCTGGAAGAG \\
\hline
\end{tabular}

F, forward; R, reverse; HBx, hepatitis B virus X protein; IL-12, interleukin-12.

modified Eagle's medium (DMEM) supplemented with $10 \%$ fetal bovine serum (FBS) containing penicillin-streptomycin antibiotics (all from Thermo Fisher Scientific, Waltham, MA, USA) at $37^{\circ} \mathrm{C}$ in a humidified incubator with $5 \% \mathrm{CO}_{2}$.

miR-21 mimics and the inhibitor were transfected into cells for upregulation or downregulation, respectively, of miR-21 expression, and an miR-21 sequence-scrambled RNA was used as the miRNA negative control (NC_miR). Pre-designed siRNAs were used to inhibit the expression of HBx. cDNA of HBx cloned into the pEGFP-N3 vector (Clontech Laboratories, Mountain View, CA, USA) was used as an $\mathrm{HBx}$ transgene (pHBx) in hepatic cells, and an empty vector (vector) was used as a negative control. The cells were transfected in vitro with the miRNAs, siRNAs and plasmids using Lipofectamine ${ }^{\circledR} 2000$ transfection reagent (Thermo Fisher Scientific) according to the manufacturer's instructions. miR-21 mimics, inhibitor, siRNAs and negative control were obtained from Biomics Biotechnologies Co., Ltd. (Nantong, China) and the sequences are shown in Table I.

Dual-luciferase reporter (DLR) assay. The cDNA of the 3'UTR region of IL-12 mRNA was constructed into the pGL3-vector (Promega Corp., Madison, WI, USA) as a dual-luciferase miRNA target expression vector (pGL3-IL-12 3'UTR wild-type) to evaluate miR-21 activity. The IL-12 3'UTR mutant vector was also constructed as the negative control (pGL3-IL-12 3'UTR mutant). HEK293 cells were seeded in a 24-well plate. After $24 \mathrm{~h}$, the cells were co-transfected with pGL3-IL-12 3'UTR wild-type or mutant vector and miR-21 mimics or NC_miR; pRL-TK (Promega Corp.) was co-transfected as internal control. Luciferase activities were measured 48-h post-transfection using the DLR assay system (Promega Corp.) according to the manufacturer's instructions.

Real-time quantitative PCR (RT-qPCR). Total RNA of the hepatocytes for mRNA detection was extracted using TRIzol ${ }^{\circledR}$ reagent (Thermo Fisher Scientific). Small RNA enriched with miRNAs was isolated using mirPremier ${ }^{\circledR}$ microRNA isolation kit (Sigma-Aldrich, St. Louis, MO, USA) according to the manufacturer's instructions.

Stem-loop RT-qPCR was performed for miR-21 expression detection as described previously (24), U6 small RNAs were used as an internal control (25). RT-qPCR was carried out using the SuperScript ${ }^{\circledR}$ III Platinum ${ }^{\circledR}$ SYBR $^{\circledR}$ Green
Table II. Sequences of the HBx-targeted siRNAs.

\begin{tabular}{|c|c|}
\hline siRNAs & Sequences $\left(5^{\prime}-3^{\prime}\right)$ \\
\hline \multirow[t]{2}{*}{ HBx_si1 } & Sense: $\quad$ GGACUCUCUGCAAUGUCAAdTdT \\
\hline & Antisense: UUGACAUUGCAGAGAGUCCdTdT \\
\hline \multirow[t]{2}{*}{ HBx_si2 } & GGGAGGAGAUUAGAUUAAAdTdT \\
\hline & Antisense: UUUAAUCUAAUCUCCUCCCdTdT \\
\hline \multirow[t]{2}{*}{ HBx_si3 } & Sense: $\quad$ GCGGGACGUCCUUUGUUUAdTdT \\
\hline & Antisense: UAAACAAAGGACGUCCCGCdTdT \\
\hline \multirow[t]{2}{*}{ HBx_si4 } & GAAUGUUGCCCAAGGUCUUdTdT \\
\hline & Antisense: AAGACCUUGGGCAACAUUCdTdT \\
\hline \multirow[t]{2}{*}{ NC_siR } & UUCUCCGAACGUGUCACGUdTdT \\
\hline & Antisense: ACGUGACACGUUCGGAGAAdTdT \\
\hline
\end{tabular}

$\mathrm{HBx}$, hepatitis B virus X protein.

One-Step RT-qPCR kit (Thermo Fisher Scientific) according to the manufacturer's instructions. The relative expression was evaluated by the $2^{-\Delta \Delta \mathrm{Ct}}$ method (26). The primer sequences are shown in Table II.

Western blot analysis. Cells were plated in 6-well plates and treated as described above. Post 48-h treatment, the cells were harvested and lysed in ice-cold cell RIPA lysis and extraction buffer (Thermo Fisher Scientific). After centrifugation for collecting, the proteins were separated by polyacrylamide gel electrophoresis and electro-transferred to polyvinylidene fluoride (PVDF) membranes (Millipore Corp., Billerica, MA, USA), and then incubated with the anti-IL-12 (1:1,000 dilution), anti-HBx antigen (1:1,000 dilution) or mouse anti-human $\beta$-actin antibody (1:5,000 dilution) (all from Abcam, Cambridge, MA, USA) as internal control. After washing with TBST, the membrane was incubated with horseradish peroxidase (HRP)-conjugated secondary antibody for $1.5 \mathrm{~h}$ at room temperature, and then washed in TBST. Finally, the specific proteins were detected with ECL substrate (Thermo Fisher Scientific).

Cell proliferation assay. Cell proliferation was measured using the Vybrant ${ }^{\circledR}$ MTT Cell Proliferation assay kit (Thermo Fisher Scientific). Hepatocytes were seeded on a 96-well plate at a concentration of $5 \times 10^{3}$ cells/well before transfection and grown to about $70 \%$ confluency for $24 \mathrm{~h}$. After treatments for $0,24,48,72$ and $96 \mathrm{~h}$, the medium was removed and replaced with $100 \mu \mathrm{l}$ of fresh culture medium, and then $10 \mu \mathrm{l}$ of the $12 \mathrm{mmol} / \mathrm{l}$ MTT stock solution was added to each well. A negative control of $10 \mu \mathrm{l}$ of the MTT stock solution added to $100 \mu \mathrm{l}$ of medium alone was included. After incubation at $37^{\circ} \mathrm{C}$ for $4 \mathrm{~h}, 100 \mu \mathrm{l}$ of the SDS-HCl $(0.01 \mathrm{~mol} / \mathrm{l})$ solution was added to each well and mixed thoroughly. The microplate was incubated at $37^{\circ} \mathrm{C}$ for $4 \mathrm{~h}$ in a humidified chamber. Each sample was mixed again and the absorbance was read at $570 \mathrm{~nm}$ using a microplate reader (BioTek, Winooski, VT, USA).

Cell apoptosis assay. Cell apoptosis following the different treatments was determined by flow cytometric (FCM) analysis 
A

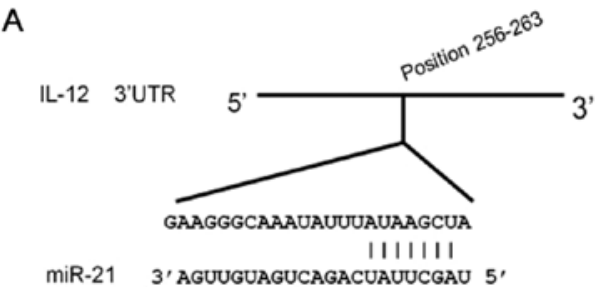

B

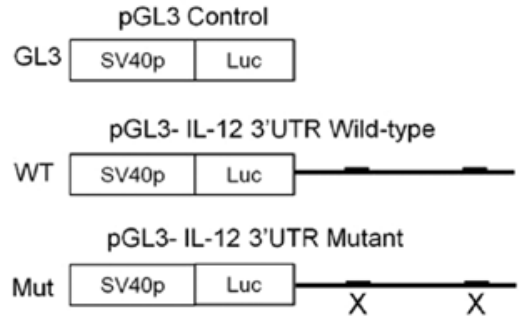

C

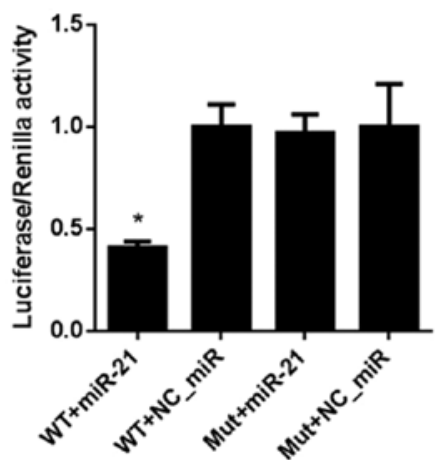

Figure 1. miR-21 targets the 3'UTR of interleukin-12 (IL-12). (A) Predicted base pairing of the mature miR-21 seed sequences in the 3'UTR of IL-12 (http:// www.targetscan.org/). (B) pGL3-IL-12 3'UTR wild-type or mutant vector for dual-luciferase reporter (DLR) assay. (C) Luciferase (normalized to Renilla) expression in human embryonic kidney 293 (HEK293) cells co-transfected with miR-21 mimic (miR-21), negative control (NC_miR) and pGL3-IL-12 3'UTR wild-type or mutant vector. ${ }^{*} \mathrm{P}<0.05$ vs. NC_miR.
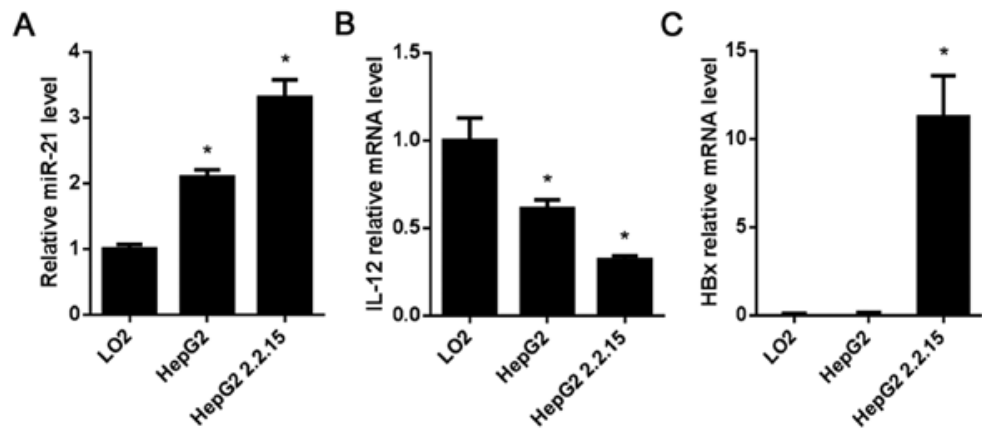

Figure 2. miR-21, interleukin-12 (IL-12) and hepatitis B virus X protein (HBx) expression in hepatocellular carcinoma (HCC) cells and normal liver L02 cells. (A) miR-21 expression level in HepG2, HepG2 2.2.15 and L02 cells. (B) IL-12 mRNA level in HepG2, HepG2 2.2 .15 and L02 cells. (C) HBx mRNA level in HepG2, HepG2 2.2.15 and L02 cells. " $\mathrm{P}<0.05$ vs. L02 cells.

with Annexin V-FITC/PI double staining. Briefly, post 48-h treatments as described above, $1 \times 10^{5}$ cells/well were harvested and washed in phosphate-buffered saline (PBS) in a 6-well plate, then re-suspended in Annexin-binding buffer, followed by incubation with Annexin V-FITC conjugate and PI for $15 \mathrm{~min}$ at room temperature. The stained cells were detected by FCM, and the results were analyzed by BD CellQuest software (BD Biosciences, Franklin Lakes, NJ, USA).

Statistical analysis. All the experiments were performed independently three times. The data are shown as mean values \pm standard deviation (SD). Statistical analyses were performed using SPSS 19.0 software, and the results were analyzed using one way ANOVA followed by post hoc test to assess statistical significance. All P-values are based on a two-sided statistical analysis and $\mathrm{P}<0.05$ was considered to indicate statistical significance.

\section{Results}

3'UTR of IL-12 mRNA is a direct target of miR-21. miRNA target predication software was used to identify the potential
miRNAs which regulate IL-12 in TargetScan database (http:// www.targetscan.org/). A putative miR-21 binding site was predicted in the 3'UTR region of IL-12 mRNA (Fig. 1A). To investigate the regulatory effects of miR-21 on IL-12, pGL3-IL-12 3'UTR wild-type and mutant vector were constructed (Fig. 1B). The results of the DLR assay showed that co-transfection of HEK293 cells with the miR-21 mimics (miR-21) and pGL3-IL-12 3'UTR wild-type or mutant vector, led to an obvious reduction in luciferase activity compared to the NC_miR (Fig. 1C). In contrast, the luciferase activity of the pGL3-IL-12 3'UTR mutant vector was not affected by upregulation of miR-21 (Fig. 1C), which further validated the direct binding between the miR-21 seed sequence and 3'UTR of IL-12 mRNA, and indicates that IL-12 is a novel direct target of miR-21.

Expression of miR-21, IL-12 and HBx in the HCC cells. miR-21 expression and IL-12 and HBx mRNA levels in the HCC cell lines HepG2 and HepG2 2.2.15 were determined by RT-qPCR. Compared with the L02 cells, the results showed that miR-21 was highly expressed in the HepG2 and HepG2 2.2.15 cells $(\mathrm{P}<0.05$; Fig. 2A), while the mRNA level of IL-12 in the 
A

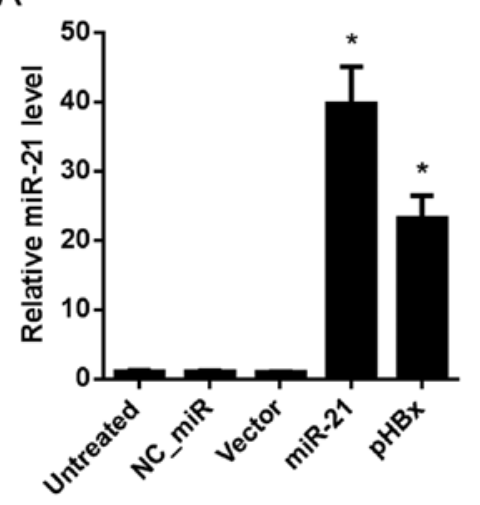

B
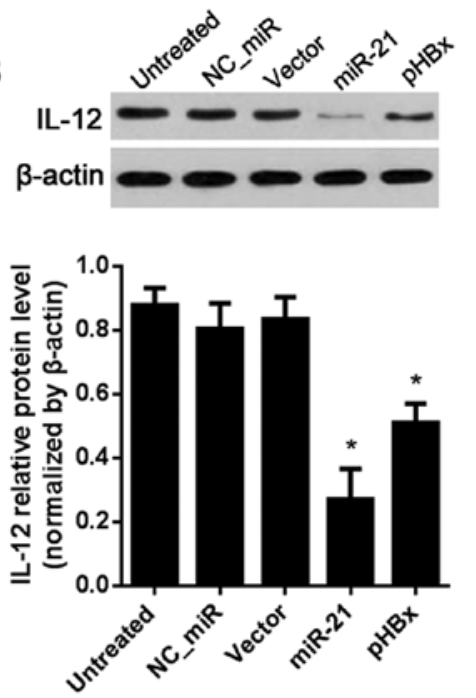

Figure 3. Interleukin-12 (IL-12) is regulated by miR-21 in L02 cells [normal liver cells with no hepatitis B virus X protein (HBx) and low miR-21]. (A) The relative miR-21 expression level was upregulated by miR-21 mimics (miR-21) and the HBx overexpression vector (pHBx). (B) The expression level of IL-12 was downregulated by miR-21 mimics (miR-21) and pHBx. ${ }^{*} \mathrm{P}<0.05$ vs. NC_miR or vector control.

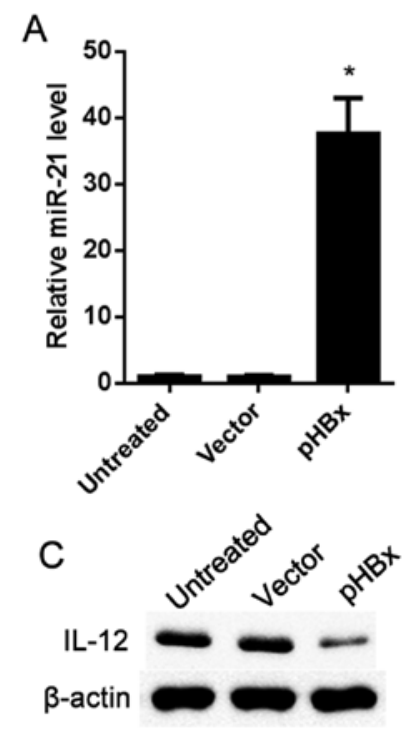

B
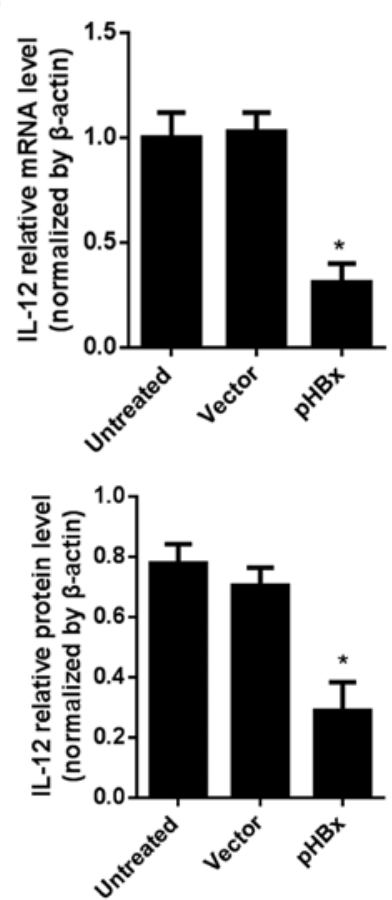

Figure 4. Interleukin-12 (IL-12) is regulated by hepatitis B virus X protein (HBx) in HepG2 cells (an HCC cell line lacking HBx expression). (A) The relative miR-21 expression level was upregulated by pHBx. (B) The mRNA level of IL-12 was downregulated by pHBx. (C) The protein level of IL-12 was inhibited by pHBx. ${ }^{*}<<0.05$ vs. vector control.

HepG2 and HepG2 2.2.15 cells was lower than that in the L02 cells ( $\mathrm{P}<0.05$; Fig. 2B), HBx was highly expressed in the HepG2 2.2.15 cells $(\mathrm{P}<0.05$; Fig. 2C).

IL-12 is regulated by miR-21 in the LO2 cells. The above results (Fig. 2) showed that miR-21 was weakly expressed in the L02 cells, and there was no HBx expression. As shown in Fig. 3, the expression level of miR-21 was significantly upregulated post-miR-21 mimic or HBx overexpression vector $(\mathrm{pHBx})$ transfection compared with that in the NC_miR or vector-treated group $(\mathrm{P}<0.05 ;$ Fig. $3 \mathrm{~A})$. The protein level of IL-12 was obviously decreased in the miR-21 and pHBx-treated group compared with the levels in the NC_miR or vector-treated group $(\mathrm{P}<0.05 ;$ Fig. $3 \mathrm{~B})$.

IL-12 is regulated by HBx in the HepG2 cells. HepG2, an HCC cell line with no HBx was used to observe the expression level of IL-12 as affected by HBx. The results showed that, compared with the vector-treated cells, miR-21 was significantly upregulated in the pHBx-treated group $(\mathrm{P}<0.05$; 
A

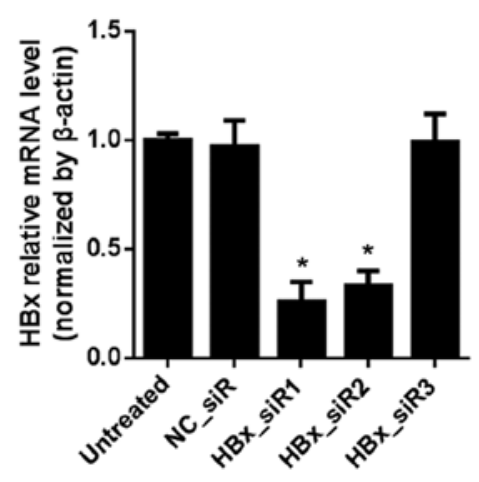

B
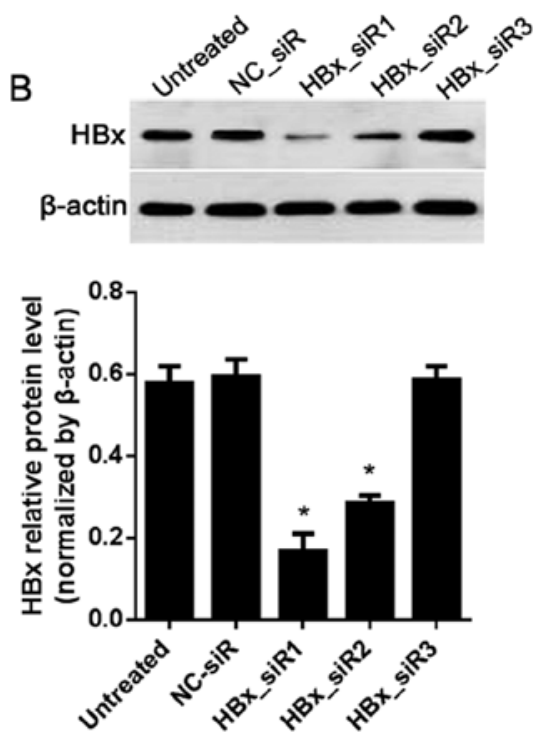

Figure 5. Inhibitory effects of hepatitis B virus X protein (HBx)-targeted siRNAs on HepG2 2.2.15 cells. (A) The mRNA expression level of HBx was inhibited by siRNAs (HBx_siR1, HBx_siR2 and HBx_siR3). (B) The protein expression level of HBx was suppressed by siRNAs. "P<0.05 vs. NC_siR.

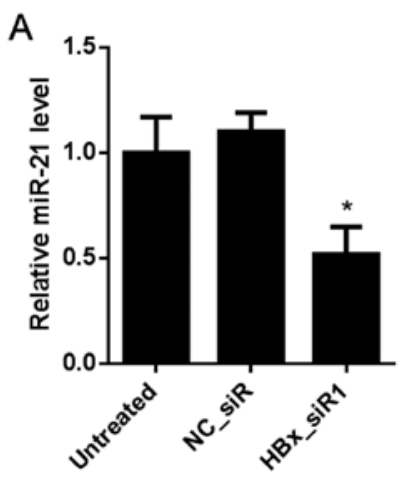

B
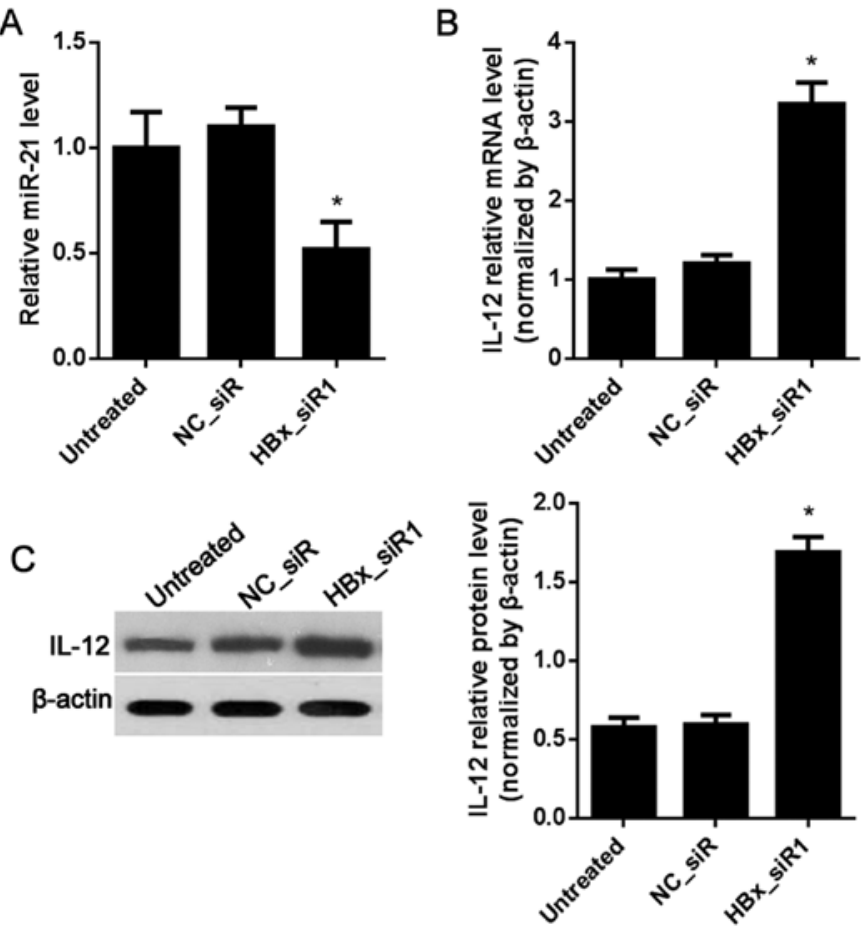

Figure 6. Interleukin-12 (IL-12) is regulated by miR-21 in HepG2 2.2.15 cells. (A) The relative miR-21 expression level was inhibited by HBx_siR1. (B) The mRNA level of IL-12 was upregulated by HBx_siR1. (C) The protein level of IL-12 was upregulated by HBx_siR1. "P $<0.05$ vs. NC_siR.

Fig. 4A). The mRNA and protein levels of IL-12 were both decreased in the pHBx-treated cells as detected by RT-qPCR and western blot analysis separately (Fig. 4B and C).

HBx is inhibited by siRNAs in the HepG2 2.2.15 cells. To clarify the correlation of HBx and/or IL-12 with miR-21, $\mathrm{HBx}$-targeted siRNAs were designed for suppression of $\mathrm{HBx}$ in the HepG2 2.2.15 cells via RNAi method. In comparison with the NC_siR-treated cells, the mRNA and protein levels of $\mathrm{HBx}$ were both inhibited by $\mathrm{HBx} \_$siR1 and HBx_siR2 $(\mathrm{P}<0.05$; Fig. 5), and HBx_siR1 was the most effective siRNA.
$I L-12$ is regulated by miR-21 in the HepG2 2.2 .15 cells. IL-12 was weakly expressed in the HepG2 2.2.15 cells as shown in Fig. 2B. The effects of the regulation of IL-12 by miR-12 when HBx or miR-21 is downregulated or upregulated were observed. Compared with the NC_siR-treated cells, miR-21 was significantly decreased in the HBx_siR1-treated cells (Fig. 6A). The mRNA and protein levels of IL-12 were both increased in the HBx_siR1-treated cells (Fig. 6B and C). To inhibit miR-21, the miR-21 inhibitor was used. The results showed that, compared with the NC_miR-treated cells, miR-21 was significantly decreased in the miR-21 inhibitor- 
A

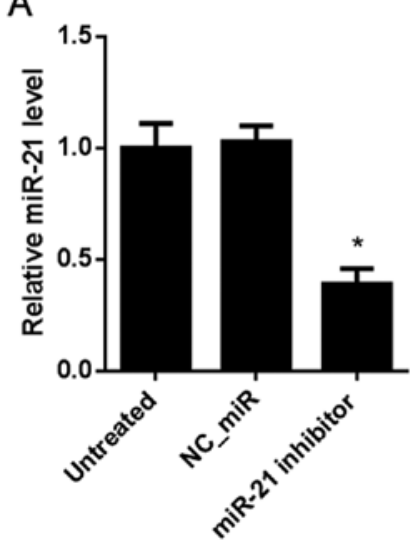

B

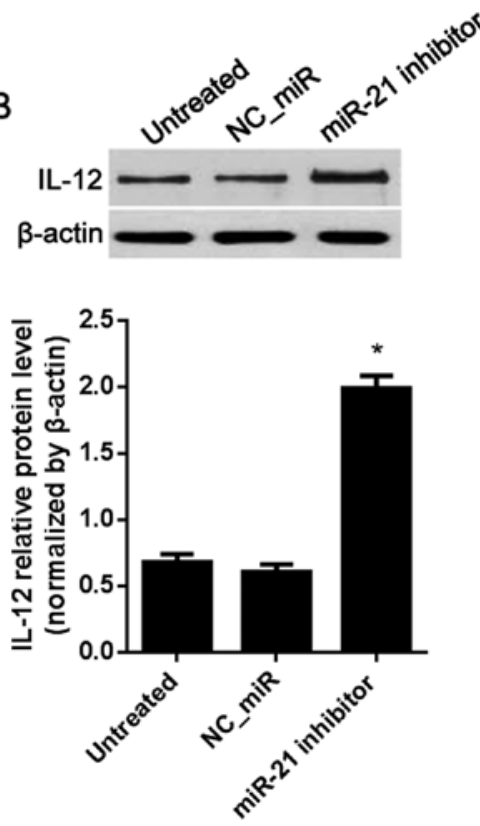

Figure 7. Interleukin-12 (IL-12) is regulated by the miR-21 inhibitor in the HepG2 2.2.15 cells. (A) The relative miR-21 expression level was inhibited by the miR-21 inhibitor. (B) The protein expression level of IL-12 was upregulated by the miR-21 inhibitor. " $\mathrm{P}<0.05$ vs. NC_miR.

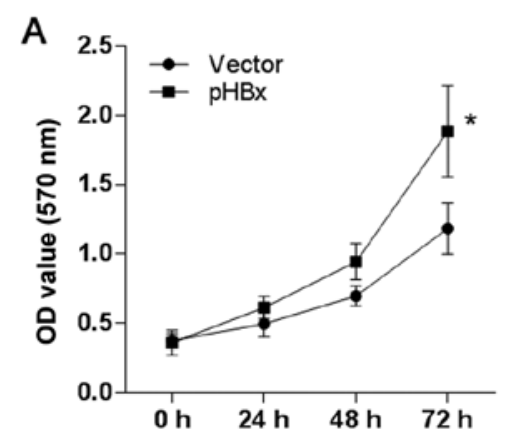

C

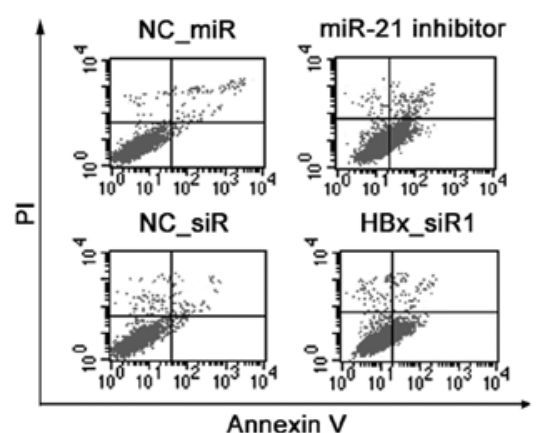

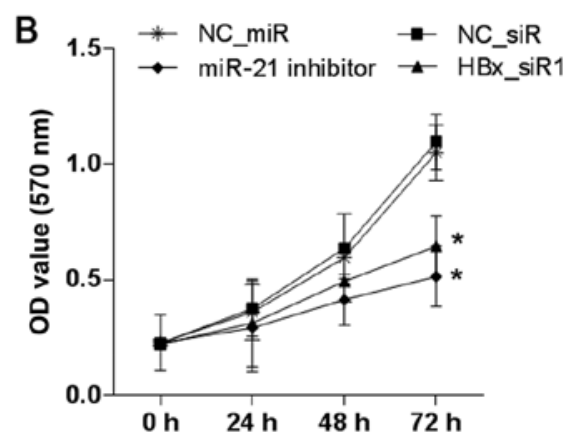

D

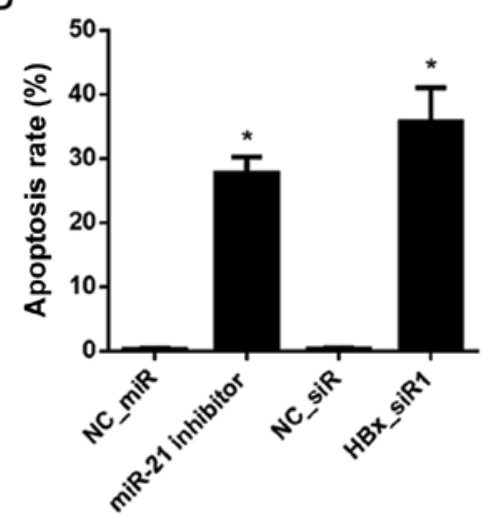

Figure 8. Proliferation and apoptosis of hepatocellular carcinoma (HCC) cells affected by hepatitis B virus X protein (HBx) or miR-21. (A) The proliferation of HepG2 cells was promoted by pHBx. "P<0.05 vs. vector control. (B) The proliferation of HepG2 2.2 .15 cells was inhibited by the miR-21 inhibitor or HBx_siR1. "P<0.05 vs. NC_miR or NC_siR. (C) Cell apoptosis was measured by flow cytometric (FCM) analysis after Annexin V-FITC/PI staining. (D) Percentage of Annexin V-FITC and PI double staining-positive cells from three independent experiments was quantified and presented as mean \pm SD. "P $<0.05$ vs. NC_miR or NC_siR.

treated cells (Fig. 7A), while the protein level of IL-12 was increased (Fig. 7B).

Proliferation and apoptosis of the HCC cells are affected by HBx or miR-21. HepG2 cells with no HBx expression and
HepG2 2.2.15 cells with high HBx expression were used to observe cell proliferation affected by HBx or miR-21 by MTT assay. Compared with the vector-treated cells, the results showed that the proliferation ability of the HepG2 cells was increased after treatment with pHBx (Fig. 8A). Compared with 
the NC_miR or NC_siR-treated cells, the proliferation ability of the HepG2 2.2.15 cells was decreased after treatment with the miR-21 inhibitor or HBx_siR1 (Fig. 8B).

In addition, the role of $\mathrm{HBx}$ or miR-21 on the apoptosis of HepG2 2.2.15 cells was examined by FCM analysis with Annexin V-FITC/PI double staining. Compared with the NC_miR or NC_siR-treated cells, treatment with the miR-21 inhibitor or HBx_siR1 resulted in a significant increase in apoptosis ( $\mathrm{P}<0.05$; Fig. $8 \mathrm{C}$ and $\mathrm{D})$.

\section{Discussion}

IL-12 used as an anticancer therapeutic in several clinical trials has demonstrated beneficial results by gene therapy, and currently antitumor studies based on IL-12 are ongoing with the key focus of reducing toxicities and side effects (27-29). Undoubtedly, cancer treatment using direct administration of IL-12 protein or IL-12 expression vector is preferred, but the production of IL-12 protein or IL-12 gene delivery is problematic to define. In the present study, we aimed to identify an optional route for the cell endogenous IL-12-inducing pathway in HCC, especially in HBX-related HCC.

$\mathrm{HBx}$ plays an important role in the development of HBV-related HCC (30). HBx has been shown to induce various signaling pathways and cellular proteins that could link HCC with HBV infection (31-33). Studies have shown that HBx protein induces the expression of oncogenic miR-21, but the molecular mechanism of the role of miR-21 in HBx-induced proliferation and apoptosis in HCC cells is still unknown. In this study, we investigated the effect of HBx on the expression of miR-21 and its role in inducing the proliferation and apoptosis induced by targeting IL-12 in HCC cells.

The results of our study showed that IL-12 is a direct target of miR-21 by binding to 3'UTR of IL-12 and DLR assay validation (Fig. 1). Furthermore, HepG2 cells with no HBx expression, and HepG2 2.2.15 cells with high expression of HBx (Fig. 2C) were used as an HCC in vitro cell model, and normal hepatocyte L02 cells were used as a control. The result of RT-qPCR showed that miR-21 was highly expressed in both HepG2 and HepG2 2.2.15 cells compared with the L02 cells (Fig. 2A), while IL-12 was weakly expressed in the two HCC cell lines $(\mathrm{P}<0.05$; Fig. $2 \mathrm{~B})$. In the L02 cells with low miR-21 and no HBx expression, the expression of IL-12 was downregulated significantly after miR-21 was increased or $\mathrm{HBx}$ was overexpressed (Fig. 3). In the HepG2 cells with no HBx, overexpression of $\mathrm{HBx}$ resulted in significant miR-21 upregulation (Fig. 4A). Upregulation of HBx resulted in a decrease in the mRNA and protein levels of IL-12 (Fig. 4B and C). In the HepG2 2.2.15 cells with high expression of HBx and high miR-21 level, HBx inhibited by siRNAs resulted in a significant decrease in miR-21 (Fig. 6A). The mRNA and protein levels of IL-12 were both increased (Fig. 6B and C). miR-21 inhibited by the miR-21 inhibitor resulted in the increase in the protein level of IL-12 (Fig. 7). These results showed that IL-12 is regulated by HBx-induced miR- 21 .

Previous studies have shown that HBx inhibits apoptosis and enhances cellular proliferation in hepatoma cells $(34,35)$, while the mechanism is unknown. Thus, further validation by MTT assay and FCM analysis showed that the proliferation ability of the HepG2 cells was increased when $\mathrm{HBx}$ was overexpressed (Fig. 8A). The proliferation ability of the HepG2 2.2.15 cells was decreased when miR-21 was inhibited by the miR-21 inhibitor or HBx_siR1 (Fig. 8B). Treatment with miR-21 inhibitor or HBx_siR1 resulted in a significant increase in HepG2 2.2.15 cell apoptosis (Fig. 8C and D).

Our study confirmed that IL-12 is a direct target of miR-21 and miR-21 can be upregulated by HBx protein in hepatic cells. A high level of HBx also resulted in the inhibition of IL-12. A high level of miR-21 resulted in a significant decrease in IL-12 expression and an increase in proliferation. Inhibition of miR-21 resulted in a significant increase in IL-12 expression and an increase in apoptosis. The results suggest that the suppression of apoptosis in HCC cells was at least partially carried out through HBx-induced miR-21 by targeting IL-12.

\section{Acknowledgements}

The present study was supported by the Youth Foundation of Nantong Health and Family Planning Commission (WQ2015018 and WQ2014005).

\section{References}

1. El-Serag HB and Rudolph KL: Hepatocellular carcinoma: Epidemiology and molecular carcinogenesis.Gastroenterology 132: 2557-2576, 2007.

2. Tan A, Yeh SH, Liu CJ, Cheung C and Chen PJ: Viral hepatocarcinogenesis: From infection to cancer. Liver Int 28: 175-188, 2008.

3. Lupberger J and Hildt E: Hepatitis B virus-induced oncogenesis. World J Gastroenterol 13: 74-81, 2007.

4. Marra M, Sordelli IM, Lombardi A, Lamberti M, Tarantino L, Giudice A, Stiuso P, Abbruzzese A, Sperlongano R, Accardo M, et al: Molecular targets and oxidative stress biomarkers in hepatocellular carcinoma: An overview. J Transl Med 9: 171, 2011.

5. McClain SL, Clippinger AJ, Lizzano R and Bouchard MJ: Hepatitis B virus replication is associated with an $\mathrm{HBx}$-dependent mitochondrion-regulated increase in cytosolic calcium levels. J Virol 81: 12061-12065, 2007.

6. Pan J, Lian Z, Wallett S and Feitelson MA: The hepatitis $\mathrm{B} \mathrm{x}$ antigen effector, URG7, blocks tumour necrosis factor alpha-mediated apoptosis by activation of phosphoinositol 3-kinase and beta-catenin. J Gen Virol 88: 3275-3285, 2007.

7. Yang B and Bouchard MJ: The hepatitis B virus X protein elevates cytosolic calcium signals by modulating mitochondrial calcium uptake. J Virol 86: 313-327, 2012.

8. Yamashita T, Budhu A, Forgues M and Wang XW: Activation of hepatic stem cell marker EpCAM by Wnt-beta-catenin signaling in hepatocellular carcinoma. Cancer Res 67: 10831-10839, 2007.

9. Soifer HS, Rossi JJ and Saetrom P: MicroRNAs in disease and potential therapeutic applications. Mol Ther 15: 2070-2079, 2007.

10. Zhang W, Lu Z, Kong G, Gao Y, Wang T, Wang Q, Cai N, Wang H, Liu F, Ye L, et al: Hepatitis B virus X protein accelerates hepatocarcinogenesis with partner survivin through modulating miR-520b and HBXIP. Mol Cancer 13: 128, 2014.

11. Bandopadhyay M, Banerjee A, Sarkar N, Panigrahi R, Datta S, Pal A, Singh SP, Biswas A, Chakrabarti S and Chakravarty R: Tumor suppressor microRNA miR-145 and onco microRNAs miR-21 and miR-222 expressions are differentially modulated by hepatitis $\mathrm{B}$ virus $\mathrm{X}$ protein in malignant hepatocytes. BMC Cancer 14: 721, 2014.

12. Li JF, Dai XP, Zhang W, Sun SH, Zeng Y, Zhao GY, Kou ZH, Guo Y, Yu H, Du LY, et al: Upregulation of microRNA-146a by hepatitis B virus X protein contributes to hepatitis development by downregulating complement factor H. MBio 6: e02459-e14, 2015. doi: 10.1128/mBio.02459-14.

13. Damania P, Sen B, Dar SB, Kumar S, Kumari A, Gupta E, Sarin SK and Venugopal SK: Hepatitis B virus induces cell proliferation via HBx-induced microRNA-21 in hepatocellular carcinoma by targeting programmed cell death protein 4 (PDCD4) and phosphatase and tensin homologue (PTEN). PLoS One 9: e91745, 2014. 
14. Li CH, Xu F, Chow S, Feng L, Yin D, Ng TB and Chen Y: Hepatitis $B$ virus $X$ protein promotes hepatocellular carcinoma transformation through interleukin- 6 activation of microRNA-21 expression. Eur J Cancer 50: 2560-2569, 2014.

15. Kobayashi M, Fitz L, Ryan M, Hewick RM, Clark SC, Chan S, Loudon R, Sherman F, Perussia B and Trinchieri G: Identification and purification of natural killer cell stimulatory factor (NKSF), a cytokine with multiple biologic effects on human lymphocytes. J Exp Med 170: 827-845, 1989.

16. Stern AS, Podlaski FJ, Hulmes JD, Pan YC, Quinn PM, Wolitzky AG, Familletti PC, Stremlo DL, Truitt T and Chizzonite R: Purification to homogeneity and partial characterization of cytotoxic lymphocyte maturation factor from human B-lymphoblastoid cells. Proc Natl Acad Sci USA 87: 6808-6812, 1990.

17. Li L, Jiang Y, Lao S, Yang B, Yu S, Zhang Y and Wu C: Mycobacterium tuberculosis-specific IL-21 $1^{+} \mathrm{IFN}-\gamma^{+} \mathrm{CD} 4^{+} \mathrm{T}$ cells are regulated by IL-12. PLoS One 11: e0147356, 2016.

18. Otani T, Nakamura S, Toki M, Motoda R, Kurimoto M and Orita K: Identification of IFN-gamma-producing cells in IL-12/IL-18-treated mice. Cell Immunol 198: 111-119, 1999.

19. Zeh HJ III, Hurd S, Storkus WJ and Lotze MT: Interleukin-12 promotes the proliferation and cytolytic maturation of immune effectors: Implications for the immunotherapy of cancer. J Immunother Emphasis Tumor Immunol 14: 155-161, 1993.

20. Yuzhalin AE and Kutikhin AG: Interleukin-12: Clinical usage and molecular markers of cancer susceptibility. Growth Factors 30: 176-191, 2012.

21. Lasek W, Zagożdżon R and Jakobisiak M: Interleukin 12: Still a promising candidate for tumor immunotherapy? Cancer Immunol Immunother 63: 419-435, 2014

22. Alatrash G, Hutson TE, Molto L, Richmond A, Nemec C, Mekhail T, Elson P, Tannenbaum C, Olencki T, Finke J, et al: Clinical and immunologic effects of subcutaneously administered interleukin-12 and interferon alfa-2b: Phase I trial of patients with metastatic renal cell carcinoma or malignant melanoma. J Clin Oncol 22: 2891-2900, 2004.

23. Gollob JA, Veenstra KG, Parker RA, Mier JW, McDermott DF Clancy D, Tutin L, Koon H and Atkins MB: Phase I trial of concurrent twice-weekly recombinant human interleukin-12 plus low-dose IL-2 in patients with melanoma or renal cell carcinoma. J Clin Oncol 21: 2564-2573, 2003.

24. Chen C, Ridzon DA, Broomer AJ, Zhou Z, Lee DH, Nguyen JT, Barbisin M, Xu NL, Mahuvakar VR, Andersen MR, et al: Real-time quantification of microRNAs by stem-loop RT-PCR. Nucleic Acids Res 33: e179, 2005.
25. Li J, Fu H, Xu C, Tie Y, Xing R, Zhu J, Qin Y, Sun Z and Zheng X: miR-183 inhibits TGF-beta1-induced apoptosis by downregulation of PDCD4 expression in human hepatocellular carcinoma cells. BMC Cancer 10: 354-363, 2010.

26. Livak KJ and Schmittgen TD: Analysis of relative gene expression data using real-time quantitative PCR and the 2(-Delta Delta C(T)) Method. Methods 25: 402-408, 2001.

27. Motzer RJ and Bukowski RM: Targeted therapy for metastatic renal cell carcinoma. J Clin Oncol 24: 5601-5608, 2006.

28. Cody JJ, Scaturro P, Cantor AB, Yancey Gillespie G, Parker JN and Markert JM: Preclinical evaluation of oncolytic $\delta \gamma(1) 34.5$ herpes simplex virus expressing interleukin-12 for therapy of breast cancer brain metastases. Int J Breast Cancer 2012: 628697, 2012

29. Parker JN, Gillespie GY, Love CE, Randall S, Whitley RJ and Markert JM: Engineered herpes simplex virus expressing IL-12 in the treatment of experimental murine brain tumors. Proc Natl Acad Sci USA 97: 2208-2213, 2000.

30. Benhenda S, Cougot D, Buendia MA and Neuveut C: Hepatitis B virus $\mathrm{X}$ protein molecular functions and its role in virus life cycle and pathogenesis. Adv Cancer Res 103: 75-109, 2009.

31. Kong GY, Zhang JP, Zhang S, Shan CL, Ye LH and Zhang XD: Hepatitis B virus $X$ protein promotes hepatoma cell proliferation via upregulation of MEKK2. Acta Pharmacol Sin 32: 1173-1180, 2011.

32. Xia LM, Huang WJ, Wu JG, Yang YB, Zhang Q, Zhou ZZ, Zhu HF, Lei P, Shen GX and Tian DA: HBx protein induces expression of MIG and increases migration of leukocytes through activation of NF-kappaB. Virology 385: 335-342, 2009.

33. Huang WC, Chen WS, Chen YJ, Wang LY, Hsu SC, Chen CC and Hung MC: Hepatitis B virus X protein induces IKK $\alpha$ nuclear translocation via Akt-dependent phosphorylation to promote the motility of hepatocarcinoma cells. J Cell Physiol 227: 1446-1454, 2012

34. Gaur AB, Holbeck SL, Colburn NH and Israel MA: Downregulation of Pdcd4 by mir-21 facilitates glioblastoma proliferation in vivo. Neuro-oncol 13: 580-590, 2011.

35. Zhou X, Ren Y, Moore L, Mei M, You Y, Xu P, Wang B, Wang G, Jia Z, Pu P, et al: Downregulation of miR-21 inhibits EGFR pathway and suppresses the growth of human glioblastoma cells independent of PTEN status. Lab Invest 90: 144-155, 2010. 